\title{
Características seminales y niveles de testosterona en carneros Pelibuey tratados con oxitocina exógena
}

\author{
Luna-Palomera, C. ${ }^{1}$; Macías-Cruz, U. ${ }^{2}$; Sánchez-Dávila F. ${ }^{3}$; Peralta-Torres, J.A. ${ }^{\text {; }}$; and Ojeda-Robertos, N.F. ${ }^{1}$
}

\begin{abstract}
'Universidad Juárez Autónoma de Tabasco, División Académica de Ciencias Agropecuarias, Tabasco. México. 2Universidad Autónoma de Baja California, Instituto de Ciencias Agrícolas, Mexicali, Baja California, México. ${ }^{3}$ Universidad Autónoma de Nuevo León, Facultad de Agronomía, Escobedo, Nuevo León, México.
\end{abstract}

PALABRAS CLAVE

Calidad seminal.

Carneros de pelo.

Cosecha de semen

Trópico húmedo.

\section{RESUMEN}

El objetivo fue determinar el efecto de la administración exógena de oxitocina (OT) previo a la obtención del semen sobre las características seminales y concentraciones séricas de testosterona en carneros Pelibuey. Se utilizaron seis carneros adultos de fertilidad probada, los cuales se trataron con 0 , 10 y $20 \mathrm{UI}$ de OT. Las tres dosis de OT se evaluaron en todos los carneros extrayendo dos eyaculados, a los 10 y 30 min postadministración del tratamiento. El volumen del eyaculado fue mayor $(p<0.01)$ con 10 UI de OT que con 0 o 20 UI de OT. La aplicación de OT (10 ○ 20 UI) no afectó (p>0.05) la motilidad en masa e individual, porcentaje de espermatozoides vivos, anormalidades primarias y concentración de espermatozoides. La concentración sérica de testosterona no varió ( $p>0.05$ ) por efecto de los tratamientos. En conclusión, la administración de OT exógena a carneros Pelibuey momentos antes de la extracción de semen con vagina artificial, solo incrementa el volumen seminal, específicamente con $10 \mathrm{UI}$.

\section{Seminal characteristic and testosterone levels in Pelibuey rams treated with exogenous oxytocin}

\section{RESUMEN}

\section{ADDITIONAL KEYWORDS}

Hair rams.

Humid tropic.

Seminal harvest.

Seminal quality.

\section{INFORMATION}

\section{Cronología del artículo.}

Recibido/Received: 15..04.2021

Aceptado/Accepted: 23.06 .2021

On-line: 15.07.2021

Correspondencia a los autores/Contact e-mail:

carlos.luna@ujat.mx

\section{INTRODUCCIÓN}

La evaluación de la capacidad reproductiva del carnero es una práctica importante para asegurar que cuenta con adecuada fertilidad, capacidad de monta y libido. En este sentido, antes de la época de apareamiento, se debe evaluar en los carneros, tanto la condición corporal como la de los órganos reproductivos, así como capacidad de servicio y calidad seminal (Lupton, 2008, p. 3553). Algunos estudios señalan que alrededor del $29 \%$ de los sementales se consideran no aptos reproductivamente, y la razón más común es por fertilidad cuestionable (43.8\%) asociada con la calidad del semen (Van Metre et al., 2012, p. 119); otras razones menos comunes son libido y capacidad de servicio reducida (Luna-Palomera et al., 2013, p. 50). Por lo anterior, se requiere la búsqueda de tratamientos hormonales que ayuden a mejorar la obtención del semen y la eficiencia reproductiva de los carneros.

La hormona neurohipofisial oxitocina (OT) junto con la vasopresina (VP) juegan un papel clave en el control de la actividad sexual (Veronesi et al., 2010, p. 464), ya que ejercen un efecto importante en la función 
eréctil y comportamiento eyaculatorio. Asimismo, estas hormonas pueden regular el transporte espermático a través de la modulación de la contractilidad en el tracto reproductivo del macho (Ivell et al., 1997, p. 255; Veronesi et al., 2010, p. 465). Estudios en ratas y carneros han descrito que la administración exógena de OT favorece la contracción de los túbulos seminíferos para impulsar la salida del esperma hacia la red testicular (Gnessi et al., 1997, p. 604; Harris and Nicholson, 1998, p. 40). Otro estudio demostró que la OT puede regular la contractibilidad epididimal basal al mismo tiempo que estimula la liberación de espermatozoides al momento de la eyaculación (Filippi et al., 2003, p. 84). Por ello, la aplicación exógena de OT en carneros podría incrementar la concentración de células espermáticas (Schenk, 2018, p. s144). A nivel de calidad seminal, la administración de 5 UI de OT en carneros incrementó el volumen, motilidad y concentración espermática a los 10 min después de su aplicación (Bozkurt et al., 2007, p. 306). En machos de otras especies domésticas como conejos (Fjellstrom et al., 1968, p. 208), toros (Berndtson and Igboeli, 1988, p. 470) y búfalos (Ibrahim, 1988, p. 7), también hay evidencia del efecto positivo que tiene la OT sobre la calidad seminal. Sin embargo, los efectos de la OT exógena en las concentraciones sanguíneas de testosterona de machos tratados son contradictorios; mientras que algunos estudios señalan una disminución (Sawada et al., 1998, p. 124) o ningún cambio (Sharpe and Cooper, 1987, p. 93), otros describen un aumento (Bozkurt et al., 2007, p. 306).

Cabe mencionar que el efecto de la OT en la calidad seminal de carneros de raza Pelibuey no ha sido evaluado. Los ovinos Pelibuey son una raza de pelo con amplia distribución en regiones tropicales y subtropicales de México y El Caribe (Aguilar-Martínez et al., 2017, p. 430). Los productores han puesto gran interés en esta raza ya que tiene una alta capacidad de adaptación a climas cálidos, no presenta estacionalidad reproductiva y es prolífica, características de gran importancia para la industria de la producción de carne ovina (Luna-Palomera et al., 2013, p. 49). En base a todo lo anterior, se planteó la hipótesis de que la aplicación exógena de OT en dosis de 10 y 20 UI vía IM ayuda a mejorar las características seminales por incrementar los niveles circulantes de testosterona. El objetivo de esta investigación fue evaluar el efecto de la aplicación exógena de OT previo a la obtención del semen con vagina artificial sobre las características seminales y concentraciones séricas de testosterona en carneros Pelibuey bajo condiciones de producción del trópico mexicano.

\section{MATERIAL Y MÉTODOS}

Condiciones experimentales y cuidado animal. El estudio se realizó en la época de verano en el rancho "El Rodeo", ubicado en la ranchería Víctor Fernández Manero, Jalapa, Tabasco, México (17 $84^{\prime \prime}$ LN, $92^{\circ} 81^{\prime \prime}$ $\mathrm{LO}$, y $10 \mathrm{msnm}$ ). Los carneros permanecieron alojados en corrales con acceso individual a comederos de madera y alimentados con una dieta integral formulada con $12 \%$ de proteína cruda y 2.0 Mcal de energía metabolizable/kg de materia seca (NRC, 2007, p. 244); adicionalmente se suministró $1.0 \mathrm{~kg}$ de forraje hidro- pónico de maíz y agua ad libitum. El cuidado y manejo de los animales se realizó en acuerdo con los criterios internos del comité de ética de la Universidad Juárez Autónoma de Tabasco, así como siguiendo la norma Oficial Mexicana NOM-062-ZOO-1999 (especificaciones técnicas para la producción, cuidado y uso de los animales de laboratorio).

Diseño experimental. Se utilizaron seis carneros Pelibuey adultos entre 2 y 4 años de edad, con un peso promedio de $65.0 \pm 1.5 \mathrm{~kg}, 3.5 \pm 0.1$ de condición corporal (escala de 5 puntos) $32.5 \pm 1.0 \mathrm{~cm}$ de circunferencia escrotal. El estudio fue realizado bajo un diseño experimental cruzado, donde el semental fue la unidad experimental y cada semental recibió los tres tratamientos que a continuación se indican: 0 (testigo), 10, y 20 UI de OT vía IM. En el caso del grupo testigo, se le administró $0.5 \mathrm{~mL}$ de solución salina (OT-0) como un efecto placebo. Los tratamientos se aplicaron en cada día de muestreo $10 \mathrm{~min}$ antes de iniciar la colecta del primer eyaculado. Los días de muestreo fueron cada tercer día acumulando seis días en total (Tabla I), cada macho se trató dos veces con un mismo tratamiento obteniendo un total de 24 eyaculados por tratamiento.

Evaluación de la calidad seminal. Se extrajeron dos eyaculados a través de vagina artificial, el primero a los 10 min y el segundo a los 30 min post-inyección del tratamiento. La vagina artificial se preparó a una temperatura de $37^{\circ} \mathrm{C}$ con agua caliente, seguido de la inyección de aire para incrementar la superficie de presión y aplicando lubricante no espermicida. A un costado de la vagina se puso una manga de látex provista de un tubo cónico graduado de $2 \mathrm{~mL}$.

Tabla I. Distribución en el tiempo de tratamientos $(0,10$ y 20 UI de OT) en carneros Pelibuey, siendo el semental fue la unidad experimental (Distribution in the time of treatments (0,10 and 20 IU of OT) in Pelibuey rams, being the stallion was the experimental unit).

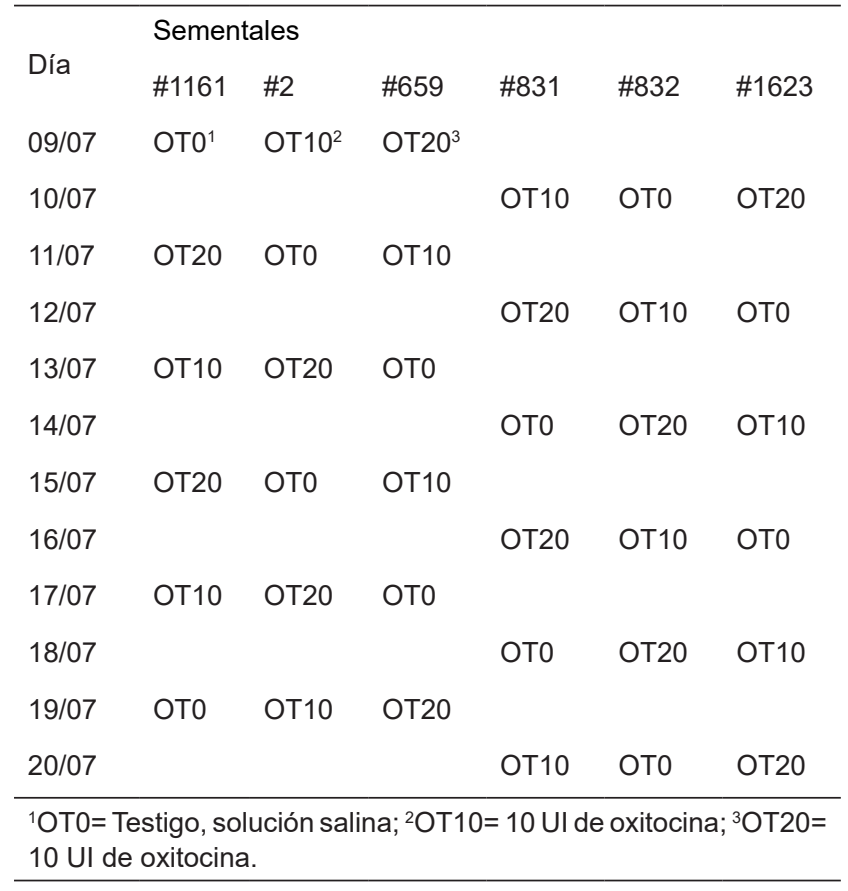


Para la evaluación de las características seminales se siguieron los criterios sugeridos por Andrade-Rocha (2003, p. 249) y Zamiri et al. (2010, p. 134). De manera directa, en el tubo cónico graduado de $2 \mathrm{~mL}$ se midió el volumen y la apariencia bajo las categorías de cremoso, lechoso y acuoso; la motilidad masal (MM) se determinó a partir de una muestra de $10 \mu \mathrm{L}$ de semen en un portaobjetos atemperado bajo un objetivo de 10X en microscopio de contraste de fases, asignando valores de 0 (motilidad nula) a 5 (motilidad en masa vigorosa). La motilidad progresiva (MP) se evaluó a partir de una muestra de $10 \mu \mathrm{L}$ de semen mezclada con $10 \mu \mathrm{L}$ de solución salina que se colocó sobre un portaobjetos atemperado cubierto y se tapó con un cubreobjetos para ser observada en el microscopio de contraste de fases usando el objetivo de 40X. La MP observada se le asignó una calificación de 0 a 100\%, de acuerdo al grado de vigor individual y movimientos progresivos en el campo.

Los porcentajes de espermatozoides vivos (ESV) y con anormalidades primarias (ANT) se determinaron en un frotis que se hizo agregando a una gota de semen eosina-nigrosina. En un campo del frotis se contaron 100 espermatozoides, los cuales se clasificaron en vivos (no teñidos) y muertos (teñidos parcial o totalmente), y posteriormente se expresó el número de espermatozoides vivos como un porcentaje del total de espermatozoides cuantificados para determinar el ESV. El mismo procedimiento se repitió para porcentaje de ANT, solo que los 100 espermatozoides se clasificaron en normales o con anormalidades (cabeza suelta y colas enrolladas o dobles). La concentración espermática por $\mathrm{mL}$ (CES) se determinó en base al número de espermatozoides por $\mathrm{mL}$ expresado en $1 \times 10^{6} \times \mathrm{mL}$. El semen se diluyó 1:200 en una solución de formalina al $0.2 \%$ y rosa de bengala al $0.5 \%$ para hacer la cuantificación en una cámara Neubauer. El conteo espermático total (CEST) se calculó multiplicando el número de espermatozoides por $\mathrm{mL}$ por el volumen del eyaculado.

Concentraciones de testosterona en sangre. Se obtuvieron muestras de sangre por venopunción de la yugular en tubos vacutainer de $5 \mathrm{~mL}$ sin anticoagulante a los 0,10 y 30 min post-administración del tratamiento. La sangre se centrifugó a temperatura ambiente $\left(30^{\circ}\right.$ C, $3000 \times \mathrm{g}$ ) por $5 \mathrm{~min}$ para obtener el suero, el cual se almacenó a $-20^{\circ} \mathrm{C}$ en viales de $2 \mathrm{~mL}$ hasta su análisis. Los niveles de testosterona se determinaron en el Laboratorio de Reproducción y Genética Animal de la División Académica de Ciencias Agropecuarias de la UJAT mediante ELISA, para lo cual se utilizó el kit comercial validado EIA-1559 (DRG Instruments $\mathrm{GmbH}$, Alemania). Los coeficientes de variación intra e inter ensayo fueron de 3.6 y $7.1 \%$, respectivamente, con una sensibilidad de $0.083 \mathrm{ng} \times \mathrm{mL}$.

Análisis estadístico. Todas las variables se analizaron con el paquete estadístico SAS (2004) usando el número de semana como una covariable. Las variables de calidad seminal se sometieron a análisis de varianza en un arreglo factorial 2 × 3 bajo un diseño completamente al azar, donde los factores principales fueron número de eyaculado y tratamiento de OT. Las concentraciones de testosterona se analizaron en mediciones repetidas en el tiempo donde el modelo incluyó los efectos fijos de tratamiento, tiempo de muestreo (0, 10 y $30 \mathrm{~min})$ $\mathrm{y}$ la interacción tratamiento $\mathrm{x}$ tiempo. Se analizaron diferentes estructuras de covarianza para ajustar el modelo, y basado en los valores más bajos de AIC y BIC se seleccionó la estructura que fue "sin estructura de covarianza". Las medias se compararon mediante diferencias mínimas significativas usando la opción PDIFF cuando fue necesario, considerando diferencias a $p \leq 0.05$. La apariencia del semen fue evaluada mediante una tabla de contingencia por Chi-cuadrado.

\section{RESULTADOS}

Los resultados de características seminales se muestran en la Tabla II Ninguna de las características seminales fue afectada $(\mathrm{p}>0.05)$ por la interacción tratamiento $x$ número de eyaculado, mientras que el tratamiento con 10 UI incrementó $(p<0.01)$ el volumen de eyaculado comparado con los tratamientos 0 y 20 UI de OT, entre los cuales no hubo diferencias ( $p>0.05$ ) para esta variable. El resto de las características seminales no fueron afectadas ( $p>0.05)$ por tratamiento. El $100 \%$ de los eyaculados presentaron aspecto cremoso.

Por otra parte, el volumen de eyaculado $(\mathrm{p}<0.01)$ y las CEST ( $\mathrm{p}<0.01)$ fueron mayores en el primer eyaculado que en el segundo. Las otras variables no fueron afectadas ( $p>0.05)$ por el número de eyaculado. Los resultados de concentración sérica de testosterona se muestran en la Figura 1, donde la interacción tratamiento $\mathrm{x}$ tiempo así como sus factores principales, no modificaron $(p>0.05)$ las concentraciones de testosterona en los carneros.

\section{DISCUSIÓN}

Los resultados obtenidos en este estudio no apoyan la hipótesis planteada, ya que el tratamiento de OT exógena minutos antes de la extracción del semen con vagina artificial no modificó las concentraciones circulantes de testosterona en los carneros, y en consecuencia tuvo reducidos efectos benéficos a nivel de características seminales. De hecho, la administración de OT solamente mejoró el volumen de eyaculado, sin afectar ninguna otra característica seminal. Esto sugiere que el tratamiento de OT a carnero de raza Pelibuey,

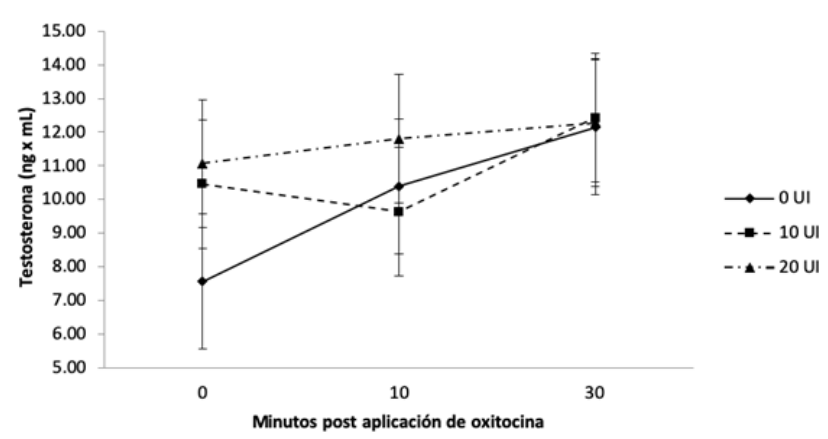

Figura 1. Concentración de testosterona (ng x mL) en carneros tratados con oxitocina exógena, previo a la aplicación (0 minutos) y post aplicación (10 y 30 minutos) (Testosterone concentration ( $\mathrm{ng} x \mathrm{~mL}$ ) in rams treated with exogenous oxytocin, prior to application ( 0 minutes) and post application (10 and 30 minutes)). 
Tabla II. Características seminales de carneros Pelibuey tratados con diferentes niveles de oxitocina (OT) (Seminal characteristics of Pelibuey rams treated with different levels of oxytocin (OT)).

\begin{tabular}{|c|c|c|c|c|c|c|c|c|c|c|}
\hline \multirow[t]{2}{*}{ Variable } & \multicolumn{4}{|c|}{ Tratamiento con OT } & \multicolumn{3}{|c|}{ Eyaculado } & \multicolumn{3}{|c|}{ Valor de $p$} \\
\hline & Testigo & $10 \mathrm{UI}$ & $20 \mathrm{UI}$ & EE & 1 & 2 & EE & OT & $\begin{array}{l}\text { Eyacu- } \\
\text { lado }\end{array}$ & OT*Eyaculado \\
\hline Volumen (mL) & $0.7^{\mathrm{b}}$ & $1.0^{\mathrm{a}}$ & $0.4^{\mathrm{b}}$ & 0.1 & $0.87^{a}$ & $0.60^{\mathrm{b}}$ & 0.06 & $<0.01$ & $<0.01$ & 0.66 \\
\hline Motilidad Masal (1-5) & $3.9^{a}$ & $4.2^{\mathrm{a}}$ & $4.2^{\mathrm{a}}$ & 0.1 & $3.98^{a}$ & $4.22^{\mathrm{a}}$ & 0.11 & 0.37 & 0.16 & 0.27 \\
\hline Motilidad Individual (\%) & $82.4^{\mathrm{a}}$ & $84.7^{\mathrm{a}}$ & $86.2^{\mathrm{a}}$ & 2.0 & $83.0^{\mathrm{a}}$ & $85.8^{a}$ & 2.35 & 0.40 & 0.06 & 0.84 \\
\hline Espermatozoides vivos (\%) & $89.5^{\mathrm{a}}$ & $90.4^{a}$ & $89.9^{a}$ & 0.8 & $0.90^{\mathrm{a}}$ & $0.88^{\mathrm{a}}$ & 0.00 & 0.57 & 0.12 & 0.19 \\
\hline Anormalidades primarias (\%) & $1.7^{\mathrm{a}}$ & $1.6^{\mathrm{a}}$ & $1.6^{\mathrm{a}}$ & 0.1 & $1.69^{\mathrm{a}}$ & $1.55^{\mathrm{a}}$ & 0.12 & 0.80 & 0.43 & 0.47 \\
\hline $\begin{array}{l}\text { Concentración por mL (x } 10^{6} \\
\left.\mathrm{~mL}^{-1}\right)\end{array}$ & $1732.1^{\mathrm{a}}$ & $1701.2^{\mathrm{a}}$ & $1521.7^{a}$ & 107.9 & $1682.5^{\mathrm{a}}$ & $1620.8^{a}$ & 88.1 & 0.33 & 0.62 & 0.35 \\
\hline $\begin{array}{l}\text { Concentración total }\left(\times 10^{6}\right. \\
\left.\mathrm{mL}^{-1}\right)\end{array}$ & $1439.9^{a}$ & $1559.2^{\mathrm{a}}$ & $1518.9^{a}$ & 220.5 & $1861.3^{a}$ & $1150.86^{b}$ & 180.4 & 0.92 & $<0.01$ & 0.45 \\
\hline
\end{tabular}

UI= unidades internacionales; EE= Error estándar; ${ }^{a, b}$ Letras diferentes entre columnas de tratamientos son diferentes estadísticamente $(p<0.05)$.

a diferencia de lo observado en carneros de otras razas (Gnessi et al., 1997, p. 561; Harris and Nicholson, 1998, p. 48; Bozkurt et al., 2007, p. 306) o machos de otra especie (Fjellstrom et al., 1968, p. 84; Berndtson and Igboeli, 1988, p. 470; Ibrahim, 1988, p. 6), no modifica la calidad seminal y sus efectos benéficos se limita a un incremento en el volumen de eyaculado.

Cabe mencionar que el volumen del primer eyaculado de los carneros se encuentra dentro del rango sugerido por Gil et al. (2002, p. 1785), quienes reportan variaciones entre 0.75 y $2.0 \mathrm{~mL}$. Los resultados encontrados con la aplicación de 10 UI de OT en el presente estudio concuerdan con los reportados por Bozkurt et al. (2007 p. 306), quienes encontraron un incremento del $38.45 \%$ en el volumen del eyaculado colectando a los 10 min después de la aplicación de 5 UI de OT por vía intravenosa. En este estudio, los carneros tratados con 10 UI de OT tuvieron 30 y 60 \% más volumen de eyaculado que los carneros del grupo testigo y los tratados con 20 UI de OT, respectivamente. Este efecto positivo podría deberse al hecho que la OT también tiene un efecto directo sobre la contracción de las colas del epidídimo, ductos deferentes y las glándulas accesorias (Poiani, 2006, p. 295; Stadler et al., 2020, p. 8), incrementando los fluidos del plasma seminal. No obstante, la dosis de 20 UI de OT no generó este efecto positivo, contrariamente disminuyó el volumen de eyaculado (numéricamente comparado con testigo y significativamente en relación al tratamiento con 10 UI); posiblemente dosis mayores que 10 UI de OT provoquen una sobreestimulación que causa un efecto antagónico en la actividad secretora de las glándulas accesorias del macho. Aunque no se encontró un reporte que indique este tipo de efectos negativos en la actividad secretora de las glándulas accesorias debido a una elevada dosis de OT.

Los resultados del incremento en el volumen del eyaculado debido a la administración de 10 UI de OT también son congruentes con los de Nicholson et al. (1999, p. 302), además ellos lo asociaron con una mejo- ra en la producción y transporte de espermatozoides, lo cual no fue evidente en el presente trabajo. Otros trabajos señalan que la administración de OT exógena en machos reproductores aumenta la concentración espermática y el volumen de eyaculado porque estimula la contracción del epidídimo, mejora el tránsito de esperma a través de los conductos espermáticos e incrementa los fluidos seminales (Voglmayr, 1975, p. 121; Palmer et al., 2004, p. 217; Schenk, 2018, p. s145). Por lo tanto, posiblemente la administración de OT a los carneros Pelibuey promovió una mayor actividad secretora de las glándulas accesorias y favoreció poco el tránsito de los espermatozoides a través de los ductos espermáticos; esto podría explicar por qué los efectos de OT se limitaron solamente a un aumento en el volumen del eyaculado en el actual estudio.

En congruencia con el efecto del número de eyaculados, Bozkurt et al. (2007 p. 306) observaron que, al realizar dos recogidas a los 60 y 120 min con respecto a la primera, el volumen descendía de $1.26 \mathrm{~mL}$ en el primero a $0.75 \mathrm{~mL}$ en el segundo y $0.35 \mathrm{~mL}$ en el tercer eyaculado. Por lo tanto, este efecto era esperado como las glándulas accesorias tienen un tiempo para recobrar totalmente su función secretora.

La falta de efecto de OT sobre MM, MP, ANT y CES también ha sido reportado en toros y humanos (Walch et al., 2001, p.658). Los valores de MP fueron satisfactorios ubicándose por arriba del 70\% de acuerdo a los criterios de evaluación usados internacionalmente (Andrade-Rocha, 2003, p. 254; Zamiri et al., 2010, p. 134) y la concentración espermática del eyaculado se ubica dentro de los criterios y concentraciones de $2.5 \mathrm{x}$ $10^{9} \mathrm{~mL}^{-1}$ reportados por Gil et al. (2002, p. 1787).

Varios trabajos (Nicholson et al., 1999 p. 302; Inaba et al., 1999, p. 430; Nozdrachev et al., 1994, p. 94) han estudiado la relación entre OT exógena y otras hormonas. Sin embargo, los resultados relativos al efecto de la OT sobre las concentraciones séricas de testosterona son controversiales, ya que algunos investigadores han informado que el tratamiento de OT reduce su con- 
centración (Inaba et al., 1999, p. 431), mientras que Nicholson et al., (1999, p. 303) y Stadler et al. (2020, p. 7) informaron que la aumenta. Por su parte, Nozdrachev et al. (1994, p. 95) documentaron que la OT no altera los niveles de testosterona en sangre. Los resultados de testosterona en este trabajo coinciden con lo encontrado por Nicholson et al. (1999, p. 303), y demuestran que, al menos en carneros Pelibuey, el tratamiento con OT exógena antes de la extracción del semen no ayuda a mejorar la calidad seminal.

\section{CONCLUSIÓN}

La aplicación de 10 UI de OT puede incrementar el volumen seminal, sin embargo, ese incremento no se refleja en una mejor calidad seminal. Como era esperado, el volumen y la cantidad de espermatozoides es mayor en el primer eyaculado que en un segundo eyaculado.

\section{AGRADECIMIENTOS}

Al rancho El Rodeo por las facilidades brindadas con los animales usados en la realización de los experimentos.

\section{BIBLIOGRAFÍA}

Aguilar-Martínez, C, Berruecos-Villalobos, J, Espinoza-Gutiérrez, B, Segura-Correa, J, Valencia-Méndez, J, \& Roldán-Roldán, A 2017 'Origin, history and current situation of Pelibuey sheep in Mexico', Tropical and Subtropical Agroecosystems, vol. 20, no. 3, pp. 429-439.

Andrade-Rocha, FT 2003 'Semen analysis in laboratory practice: An overview of routine tests', Journal Clinical Laboratory Analysis, vol. 17 , no. 6, pp. 247-58.

Berndtson. WE \& Igboeli, G 1988 'Spermatogenesis, sperm output and seminal quality of $H$ olstein bulls electroejaculated after administration of oxyłocin', Journal Reproduction and Fertility, vol. 82, no. 2, pp. 467-75.

Bozkurt, T, Turk, G \& Gur, S 2007 'Effects of exogenous oxytocin on serologic and seminal steroids and semen characteristics in rams', Turkish Journal Veterinary Animal Science, vol. 31, no. 5, 303-9.

Filippi, S, Vignozzi, L, Vannelli, GB, Ledda, F, Forti, G \& Maggi M 2003 'Role of oxytocin in the ejaculatory process', Journal of Endocrinological Investigation, vol. 26, no. 3, pp. 82-6.

Fjellstrom, D, Kihlstrom, JE \& Melin, P 1968 'The effect of synthetic oxytocin upon seminal characteristics and sexual behaviour in male rabbits', Journal of Reproduction Fertility, vol.17, no. 1, pp. 207-9.

Gil, J, Rodriguez-Irazoqui, M, Söderquist, L \& Rodriguez-Martinez, H 2002 'Influence of centrifugation or low extension rates prefreezing on the fertility of ram semen after cervical insemination'. Theriogenology, vol. 57, no. 7, pp. 1781-92.

Gnessi, L, Fabbri, A \& Spera, G 1997 'Gonadal peptides as mediators of development and functional control of the testis: an integrated system with hormones and local environment', Endocrine Reviews, vol. 18, no. 4, pp. 541-609.

Harris, GC \& Nicholson, HD 1998 'Characterization of the biological effects of neurohypophysial peptides on seminiferous tubules', The Journal of Endocrinology, vol. 156, no. 1, pp. 35-42.
Ibrahim, MA 1988 'Influence of oxytocin and prostaglandin on semen characteristics and process of ejaculation in buffalo bulls', Acta Veterinaria Hungarica, vol. 36, no. 1-2, pp. 3-10.

Inaba, T, Nakayama, Y, Tani, H, Tamada, H, Kawate, N \& Sawada, T 1999 'Oxytocin gene expression and action in goat testis', Theriogenology, vol. 52, no. 3, pp. 425-34.

Ivell, R, Balvers, M, Rust, W, Bathgate, R \& Einspanier, A 1997 'Oxyłocin and male reproductive function', Advances in Experimental Medicine and Biology, vol. 424, pp. 253-264.

Luna-Palomera, C, Aguilar-Cabrales, JA, Peralta-Torres, JA \& VelázquezMartínez, JR 2013 'Efecto del aceite de palma sobre el crecimiento y capacidad reproductiva de carneros de pelo púberes', Archivos de Zootecnia, vol. 62, no. 237, pp. 45-52.

Lupton, CJ 2008 'Impacts of animal science research on United States sheep production and predictions for the future', Journal of Animal Science, vol. 86, no. 11, pp. 3252-74.

National Research Council (NRC) 2007 Nutrient Requirements of Small Ruminants: Sheep, Goats, Cervids, and New World Camelids', Washington DC, The National Academies Press, pp. 347 https://doi. org/10.17226/11654.

Nicholson, H, Parkinson, T \& Lapwood, K 1999 'Effects of oxytocin and vasopressin on sperm transport from the cauda epididymis in sheep', Journal Reproduction and Fertility, vol. 117, no. 2, pp. 299-305.

Nozdrachev, AD, Kovalenk, RI, Chernysheva, MP \& Semenova, EP 1994 'The effect of the intraventricular administration of oxytocin on the functional activity of the epiphysis, adrenals and gonads and on the behavior of rats', Rossiiskii fiziologicheskii zhurnal imeni I.M. Sechenova, vol. 80, pp. 88-97.

Palmer, CW, Amundson, SD, Brito, LFC, Waldner, CL \& Barth, AD 2004 'Use of oxytocin and cloprostenol to facilitate semen collection by electroejaculation or transrectal massage in bulls', Animal Reproduction Science, vol. 80, no. 3-4, pp. 213-23.

Poiani, A 2006 'Complexity of Seminal Fluid: A Review', Behavioral Ecology and Sociobiology, vol. 60, no. 3, pp. 289-310. doi: $10.2307 / 25063816$

SAS 2004 'Statical Analysis System', Institute Inc/STAT® 9.1 User's Guide. Cary, NJ.

Sawada, T, Uemura, K, Tamada, H, Inaba, T \& Mori J 1998 'Effects of oxytocin and prostaglandin $\mathrm{F} 2 \mathrm{a}$ on androgen production of adult rat testis in vivo', Prostaglandin and Other Lipid Mediator, vol. 55, no. 2-3, pp. 121-26.

Schenk, JL 2018 'Review: Principles of maximizing bull semen production at genetic centers', Animal, vol. 12, no. s1, pp. s142-s147.

Sharpe, RM \& Cooper, I 1987 'Comparison of the effects on purified Leydig cells of four hormones (oxytocin, vasopressin, opiates and LHRH) with suggested paracrine roles in the testis', The Journal of Endocrinology, vol. 113, no. 1, pp. 89-96.

Stadler, B, Whittaker, MR, Exintaris, B \& Middendorff, R 2020 'Oxytocin in the male reproductive tract; The therapeutic potential of oxytocinagonists and-antagonists', Frontiers in Endocrinology, 11, pp. 1-22. https://doi.org/10.3389/fendo.2020.565731

Van Metre, DC, Rao, S, Kimberling, CV \& Morley, PS 2012 'Factors associated with failure in breeding soundness examination of Western USA rams', Preventive Veterinary Medicine, vol. 105, no. 1-2, pp. 118-26. 
Veronesi, C, Tosi, U, Villani, M, Govoni, N, Faustini M., Kindahl, H, Madej, A \& Carluccio A 2010 'Oxyłocin, vasopressin, prostaglandin F2a, luteinizing hormone, testosterone, estrone sulfate, and cortisol plasma concentrations after sexual stimulation in stallions', Theriogenology, vol. 73, no. 4, pp. 460-67.

Voglmayr, JK 1975 'Output of spermatozoa and fluid by the testis of the ram and its response to oxytocin', Journal of Reproduction and Fertility, vol. 43, no. 1, pp. 119-22.
Walch, K, Eder, R, Schindler, A \& Feichtinger, W 2001 'The effect of single-dose oxytocin application on time to ejaculation and seminal parameters in men', Journal of Assisted Reproduction and Genetics, vol. 18, no. 12, pp 655-59.

Zamiri, MJ, Khalili, B, Jafaroghli, M \& Farshad, A 2010 'Seasonal variation in seminal parameters, testicular size, and plasma testosterone concentration in Iranian Moghani rams', Small Ruminant Research, vol. 94, no. 1-3, pp. 132-36. 